Editorial

\title{
Introduction to a New Journal: Corrosion and Materials Degradation
}

\author{
Raman Singh \\ Departments of Mechanical \& Aerospace Engineering and Chemical Engineering, Monash University, \\ Melbourne, VIC 3800, Australia; raman.singh@monash.edu
}

Received: 30 March 2018; Accepted: 30 March 2018; Published: 2 April 2018

check for updates

Scope:

Corrosion is among the most common forms of materials degradation which poses enormous challenges across industries, and can even impact our health. For example, degradation of artificial implants (such as hips and knees) due to corrosive body fluid and wear may necessitate their premature replacement, which alone can cost any economy enormously. Corrosion products of degrading implants can also be toxic. Corrosion is also invariably a concern for infrastructure (buildings, roads, and bridges).

The age-old and vexing problem of corrosion continues to attract research attention, not only because it continues to cost our society very dearly, but also for its bearings on the application of new and critical technologies. For example, whether it is the use of magnesium alloys as novel biodegradable temporary implants, or material for nuclear waste containers, desalination plants, or durable electronic devices, corrosion often stands out as a non-trivial challenge. It is true that circumventing corrosion in such critical applications is technologically challenging, socially fulfilling, as well as commercially attractive, but it is equally true that a durable solution calls for a disruptive approach, which, in itself, is a non-trivial challenge (given the age-old nature of the discipline). On the other hand, given the very large economic losses caused by corrosion, the emergence of a new material often triggers an interest in its applicability for corrosion resistance. Most recent examples include ultrathin coatings of graphene and hexa-boron nitride. Corrosion, and the science and technology of its mitigation, therefore, is like an antique - old but fascinating.

The proposed journal has the core objective of providing a new platform for dissemination of disruptive and novel approaches to corrosion mitigation for commercially-attractive exploitation, while also promoting the cutting-edge advancements of the traditional approaches of the discipline.

Inaugural issues, i.e., the first two issues of the new journal, will be the compilations of the topmost class reviews that are aimed to be accomplished only upon invitation to the leading corrosion researchers and technologists on the topics, but not limited to those listed below:

(1) Salient Features of Fundamentals of Corrosion;

(2) Electrochemistry of Corroding Interfaces;

(3) Advanced Coatings (such as Graphene);

(4) Non-destructive Evaluation of Corrosion;

(5) Corrosion of Body Implants;

(6) Role of Nano- and Microstructure in Corrosion;

(7) Corrosion-assisted Cracking;

(8) Corrosion and Corrosion-assisted Fracture of Aerospace Structures;

(9) Corrosion of Renewable/Modern Energy Systems;

(10) Corrosion of Traditional Energy Systems; 
(11) Corrosion of Nuclear Energy Systems;

(12) Corrosion of Light Metals and Alloys;

(13) Corrosion of Weldments;

(14) Corrosion in Petroleum, Oil, and Gas Systems; and

(15) Corrosion in Water Systems.

\section{Background and Invitation:}

Corrosion of engineering alloys, and its mitigation measures, continue to cost dearly ( 4\% of GDP of any developed economy, which translates to an annual loss of $\sim \$ 250$ billion to the USA). Traditional approaches, such as the use of corrosion resistance alloys and coatings, have brought about significant mitigation of the age-old problem of corrosion. Alloys with the highest corrosion resistance perform on the principle of their inherent ability to develop a corrosion-resistant surface film of chromium oxide. However, chloride ions (i.e., abundantly present in sea-water) can disrupt this protective oxide film not only in lesser alloys, but even in the case of the alloys with the highest resistance when the corrosive environment is very aggressive. Hence, the traditional mitigation strategies, such as the one based just on increasing the chromium content of alloys, have not always succeeded in providing durable mitigations. However, a durable corrosion resistance is still a non-trivial challenge in some critical applications, such as where highly-corrosive solutions are handled (e.g., concentrated chloride solutions in desalination plants), where corrosion resistance is required for very long durations (e.g., nuclear waste containers), or where corrosion can cause serious health problems (such as the degradation of human implants).

With the above background, the first two issues of the new journal will be the compilations of reviews only upon invitation to the leading experts in the areas listed in the scope. 